
\title{
25 Research Soure \\ Traditionally brewed rice beverage alters gut microbiota and metabolites in mice
}

\section{Bhuwan Bhaskar}

Institute of Advanced Study in Science and Technology

Atanu Adak

Institute of Advanced Study in Science and Technology

\section{Santanu Das}

Institute of Advanced Study in Science and Technology

Mojibur Khan ( $\nabla$ mojibur.khan@gmail.com )

Institute of Advanced Study in Science and Technology (IASST) https://orcid.org/0000-0002-6909-

1479

Original article

Keywords: Gut microbiota, Histology, Metabolites, Rice beverage, Next generation sequencing

Posted Date: February 24th, 2021

DOI: https://doi.org/10.21203/rs.3.rs-221115/v1

License: (c) (1) This work is licensed under a Creative Commons Attribution 4.0 International License.

Read Full License 


\section{Abstract}

The study is an attempt to validate the effects of fermented rice beverage on gut microbiota and health, using mouse model. Rice beverage, soluble and insoluble fractions were fed to the animals for 30 days. Gut bacteria and metabolites were studied on 0th and 30th days of treatments. Next generation of sequencing of fecal metagenomic 16S rDNA indicated changes in abundance of 17 bacteria in the rice beverage treatment followed by 11 and 7 respectively in the soluble and insoluble fractions. Fecal metabolites of treatments as determined by GC-MS analyses, clustered differently based on Partial least square discriminant analysis. There were 253 negative and 160 positive correlations between relative abundance of fecal metabolites with bacteria. Histological analysis showed that microbial changes and higher plasma endotoxin levels in insoluble fraction treated group were accompanied by increased lymphocytes infiltration in lamina propia and cytoclasis of goblet cells as demonstrated by intestinal histology.

\section{Key Points}

- The study hypothesized to elucidate the effect of a fermented rice beverage on gut microbiota and metabolites

- Different fractions of rice beverage led to changes in gut microbiota in mice

- Treatment wise differences were observed in the fecal metabolites

\section{Introduction}

The microbes residing in the intestine, collectively called 'gut microbiota' influence health and well-being of the host. Some important functions of the gut microbiota include metabolic functions and energy salvage, production of short-chain fatty acids (SCFAs) and vitamins, strengthening the immune system and preventing colonization of pathogens (Guarner and Malagelada 2003). The microbes get colonized into the host gradually after birth through complex process of host-microbe interactions (Foster et al. 2017). Disturbance in the microbiota composition leads to a condition called dysbiosis which implicates diseased conditions such as allergy, inflammatory bowel disease, obesity, diabetes and cancer (Clemente et al. 2012). Various factors such as diet, genetics, ethnicity, environmental conditions etc. are known to affect the microbiota and its functions, of which diet plays the dominant role (Dehingia et al. 2015). In recent years, extensive studies have unveiled understanding the relationships between gut microbiota and diet (Shibayama et al. 2018). Interestingly, fermented foods and beverages are gaining global popularity not only because of the organoleptic properties but also in terms of health benefits. They confer several benefits to human health and also increase the population of probiotic bacteria in the gut (Marco et al. 2017). The microbes present in these foods modulate the composition of host gut microbiota. Derrien and van Hylckama (2015) described three possible mechanisms by which these microbes affect the host, viz. (1) trophic interactions (e.g. production of SCFA), (2) direct inhibition of 
pathogens (by producing bacteriocins, lowering the $\mathrm{pH}$ etc.) and (3) indirectly by production of hostderived molecules (IgA, mucins etc.) (Derrien and van Hylckama Vlieg 2015).

Rice beverage fermentation is carried out using a traditional plant-based starter cake containing their indegenous microorganisms (Jeyaram et al. 2008). During the fermentation process, rice starch is broken down into sugars which are further converted into alcohol, acids and other metabolites by the action of different microbes. Studies on biochemical analyses of the beverage detected the presence of microbial originated organic acids such as lactic acid, amino acids and prebiotic sugars like raffinose and trehalose (Das et al. 2014). Antimicrobial activities against common pathogens and antioxidant properties based on free radical scavenging have also been studied by different groups (Roy et al. 2012; Ghosh et al. 2015). Besides regular consumption, it is traditionally acclaimed to have health modulating effects for insomnia, headache, body ache, inflammation, diarrhea and urinary problems (Das et al. 2012). The present study aimed to evaluate the effect of fermented rice on the gut bacterial profile and fecal metabolites in mouse model.

\section{Materials And Methods}

\section{Fermentation of rice}

Rice was fermented following the traditional knowledge of the Tiwa community of Assam, India. The starter cake was prepared in the laboratory as described in the patent filed earlier (Khan et al. 2017). One $\mathrm{kg}$ of rice was boiled to cook and allowed to cool down to room temperature. A $30 \mathrm{gm}$ of the starter cake was crushed to make homogenous powder and mixed with the rice and kept for fermentation at $30^{\circ} \mathrm{C}$. After fermentation, the mass was filtered and the liquid (RB) was harvested and centrifuged at $5000 \mathrm{rpm}$ for $10 \mathrm{~min}$ to separate the supernatant containing the soluble fraction (SF) and the pellet was resuspended in sterile water upto the initial volume, denoted as insoluble fraction (IF).

\section{Mouse experiment}

Five weeks old, male Swiss albino mice ( $\mathrm{n}=6$ per group) were used for the study. Animals were housed in polypropylene cages (Cat. no. 041000 Tarsons, India) and standard rodent chow diet (Table 1), drinking water and a 12-hour day-night cycle was maintained throughout the experiment. Dietary intakes per cage and body weight per animal were monitored during the experimental period. After a seven-day acclimatization period, the animals were divided randomly into three treatments respectively as, control (CT), rice beverage (RB), insoluble fraction (IF) and soluble fraction (SF). A 200-230 $\mu$ l of RB, IF and SF were administered whereas the controls $(\mathrm{CT})$ received sterile water using gavage. The dosage was equivalent to the reported human intake of the beverage based on their body weight to make a consistent gavage volume (Das et al. 2014). The treatments were performed once daily in at a fixed time. Fecal samples of individual animals were collected on $0^{\text {th }}$ and $30^{\text {th }}$ days of treatments and stored at $-80^{\circ} \mathrm{C}$ immediately. Animals from each group were sacrificed and intestines were collected for histology. All experiments were conducted following the guidelines of the Committee for the Purpose of Control and 
Supervision of Experiments on Animals (CPCSEA), Ministry of Environment, Forest and Climate change, Government of India and approved by institutional animal ethical committee (approval no. IASST/IAEC/2016-17/07).

\section{Isolation of fecal metagenomic DNA and next generation sequencing (NGS) analyses}

Metagenomic DNA was extracted from the fecal samples of three animals from each treatment group using QIAGEN DNA Stool Mini-Kit (QIAGEN, Hilden, Germany) and subjected to NGS analysis with sequencing service provider Macrogen Inc. (Seoul, Republic of Korea). PCR amplification of metagenomic DNA was performed using 341F-805R primer pairs spanning the $V_{3}-V_{4}$ region of the $16 S$ rDNA gene. NGS library was prepared using Nextera XT library preparation kit according to the Illumina MiSeq protocol (Illumina Inc., 2017). Sequencing was carried out in an Illumina MiSeq machine (MiSEq 2500) following 2 $\times 300 \mathrm{bp}$ paired-end chemistry with the multiplexed pooled samples. Analysis of NGS data was performed using the QIIME2 (v 2018.6) pipeline using various available plugins. For denoising, filtering, merging and removal of chimera from raw llumina reads, the DADA2 plugin was used. Sequences having a minimum Phred score of 25 were considered for analysis and hence truncated at $270 \mathrm{bp}$ for a proper merging of forward and reverse sequences. Sequences were aligned with MAFFT and a phylogenetic tree was constructed using FastTree2 (Katoh and Standley 2013). The most recent SILVA 132 database, which contains taxonomic information for the domains of bacteria was used for the assignment of taxa by training a Naïve Bayes classifier targeting the $V_{3}-V_{4}$ region of the $16 \mathrm{~S}$ rDNA gene (Balvočiute and Huson 2017).

\section{Alpha and beta diversity indices of gut microbiota}

Alpha diversity, a measure of species richness and evenness within a single sample, was used to compare the taxa diversity between the fecal samples of different treatments. Pielou's Evenness, Shannon's diversity, Faith's phylogenetic diversity and observed species richness were estimated. Beta diversity was estimated to measure the changes in the composition of bacterial communities between different treatment groups. Jaccard and Bray-Curtis indices were used respectively for qualitative and quantitative measurement of community differences whereas UniFrac measures were used to determine the phylogenetic distances between the sets of taxa.

\section{Correlation between bacterial genera}

Spearman's rank correlation analysis was performed in R (v 3.6.1) using the Hmisc package. Bacteria with an abundance of $1 \%$ and more were selected $(n=22)$ and the interactions among those were computed by Spearmann's rank correlation. Visualization was performed with corrplot package ( $v 0.84)$.

\section{Non-targeted fecal metabolite profiling by Gas Chromatography Mass Spectrometry (GC-MS) analysis and their association with different bacteria}


Fecal sample preparations and the GC-MS run program were followed as per protocol described elsewhere (Weir et al. 2013). The analysis was carried out in a Shimadzu GC 2010 Plus-triple Quadrupole (TP-8030) GC-MS/MS system fitted with EB-5MS column (length: $30 \mathrm{~m}$, thickness: $0.25 \mu \mathrm{m}$ and ID: 0.25 $\mathrm{mm}$ ). The mass spectra were matched in the National Institute of Standards and Technology library, USA. Noisy peaks and columns bleeds were removed before data analysis. Partial least squares-discriminant analysis (PLS-DA) of the fecal metabolites on $30^{\text {th }}$ day of treatment was performed using Metaboanalyst (Chong et al. 2019). Correlation network between bacterial genera and the metabolites was visualized using Cytoscape 3.6.1 software.

\section{Assay of plasma endotoxin}

Endotoxin concentration in mice plasma was determined with Pierce LAL Chromogenic Endotoxin Quantitation Kit (Thermo Fisher Scientific) following manufacturer's instructions. The standard linear graph was prepared using Escherichia coli endotoxin with a range from 0.1-1.0 EU/ml. A $100 \mu \mathrm{l}$ of plasma sample was used for assay and the endotoxin concentration was expressed as $\mathrm{EU} / \mathrm{ml}$.

\section{Histology of intestine}

About 2-3 cm of distal part of ileum was isolated and fixed in Carnoy's solution (60\% ethanol, $30 \%$ chloroform and $10 \%$ glacial acetic acid). After 2 hrs of fixation, tissues were dehydrated in graded alcohol (50-100\%) and finally cleared in xylene. The clear tissues were embedded in paraffin and were cut into 3-5 $\mu \mathrm{m}$ thickness using microtome (Leica RM 2235, Wetzlar, Germany). The tissue sections were stained with haematoxylin and eosin (HE) and the villus length was measured from the base to the top of the villus, and the crypt depth was measured between the crypt-villus junction and the base of the crypt (Zhou et al. 2011). Examination and computer analysis of the histological sections were performed without knowledge of the origin of tissue samples.

\section{Statistical analyses}

To determine the association of fecal metabolites and bacteria, analysis was performed in $\mathrm{R}$ package $\mathrm{V}$ 3.5.1 where rcorr and corrplot packages were used respectively for the Spearman's rank correlation analysis and visualization of the correlation matrix (Wei et al. 2017). Comparison of relative abundance data of selected bacterial genera at $0^{\text {th }}$ and $30^{\text {th }}$ days of treatment was performed using the MannWhitney U test within SPSS (IBM SPSS 20, SPSS Inc, Chicago, IL). Differences in beta diversity indices were measured by ANOSIM. Kruskal-Wallis $\mathrm{H}$ test was performed to determine the differences in alpha diversity indices. Spearman's correlation analysis was performed within R (v 3.6.1) using the Hmisc package to determine the interactions between different bacteria (abundance $\geq 1 \%$ ). One-way ANOVA was performed to determine the differences in the plasma endotoxin levels within SigmaPlot (Systat Software Inc., USA).

\section{Results}




\section{NGS analysis of fecal metagenomic DNA and bacterial diversity}

Rarefaction curves suggested that an even sampling depth of 11822 sequences was sufficient to analyze diversity indices. No changes were observed in Shannon's diversity, Faith's PD and Observed OTUs in the treatment groups, at $0^{\text {th }}$ and $30^{\text {th }}$ days of treatment. The soluble fraction treated group showed a significant decrease in Pielou's evenness from 0.87 to $0.83(p=0.04)$ after 30 days of treatment (Table 2). ANOSIM indicated distinct distances among the treatment groups; Bray-Curtis ( $p=0.009$ and $\mathrm{R}=0.176)$, Jaccard's ( $p=0.044$ and $\mathrm{R}=0.116)$, unweighted UniFrac $(p=0.013$ and $\mathrm{R}=0.191)$ and weighted UniFrac $(p=0.003$ and $\mathrm{R}=0.264)$ (Fig. 1).

Based on mean frequency (\% abundance), the top 10 prevalent taxa were Prevotellaceae UCG-001 (13.06\%), Lachnospiraceae NK4A136 group (10.19\%), Lactobacillus (7.29\%), Lachnospiraceae (unassigned genus) (6.80\%), Bacteroides (5.38\%), uncultured bacterium from Muribaculaceae family (4.50\%), Alistipes (4.14\%), Ruminococcaceae UCG-014(3.32\%), Helicobacter (3.08\%) and uncultured bacterium from Lachnospiraceae family (2.91\%). As determined by Mann-Whitney U test, a maximum shift was observed in abundance of 17 bacterial genera in the RB followed by the SF treated group showing a significant difference in 11 bacterial genera between $0^{\text {th }}$ and $30^{\text {th }}$ days of treatments (Table 3 ). In the RB treated group, Muribaculaceae, Alistipes, Prevotellaceae UCG-001, Alistipes, Candidatus, Anaeroplasma and two genera belonging to unclassified Ruminococcaceae family NK4A214 group and UBA1819 increased significantly after 30 days of treatment $(p<0.05)$. In the same group, bacterial genera Bacteroides, Helicobacter, Lactobacillus, Clostridiales; D Family XIII, Lachnoclostridium, Lachnospiraceae, Anaerotruncus, Betaproteobacteriales, and Rodentibacter decreased significantly $(p<0.05)$. In the SF treated group, Bacteroides, Alistipes, Leptotrichia and an uncultured bacterium were found to decrease significantly $(p<0.05)$. Also, four unclassified bacteria, two out of which belonged to Clostridiales vadin BB60 and others belonging to Bacteroidales and Desulfovibrionaceae decreased $(p<0.05)$. In the same group, Prevotellaceae UCG-001 and Mycoplasma increased $(p<0.05)$. In the IF treated group, an increase was observed in the Rikenellaceae and Christensenellaceae R-7 group $(p<0.05)$, whereas unclassified bacteria belonging to Muribaculaceae, Prevotellaceae, and Dorea, Lachnoclostridium and Desulfovibrio decreased significantly $(p<0.05)$.

\section{Correlation between different bacteria}

An uncultured bacterium from Desulfovibrionaceae showed correlation with maximum number of bacteria $(p<0.05)$ showing a negative correlation with 5 and positive correlations with 11 bacteria. On the other hand, Helicobacter had negative correlation with Prevotellaceae UCG.001 ( $p=0.017$ and R= -0.507 ) and positive correlation with Lachnospiraceae $(p=0.0021$ and $\mathrm{R}=-0.487$ ) (Fig. 2).

\section{Fecal metabolites after 30 days of treatment and their correlations with bacteria}

A total of 70 compounds were detected with peak area greater than $0.1 \%$. Of these, 25 compounds were majorly categorized as organic acids, 8 sugar alcohols, 7 amino acids and 6 short chain fatty acids. Others included inorganic compounds and acids sugars and amino acid derivatives. Multivariate 
statistical analysis was performed for the fecal metabolites using partial least squares discriminant analysis (PLS-DA) test among the treatment groups. PLS-DA plot indicated clustering of the treatment groups based on the metabolite profiles. Moreover, IF treated group clustered with the controls (Fig. 3). Positive correlations were observed between Ruminococcaceae, with octadecanoic acid (Stearic acid) $(p=0.000$ and $\mathrm{R}=0.836)$ and oleic acid $(p=0.000$ and $\mathrm{R}=0.836)$ and negative correlations with galactose $(p=0.000$ and $\mathrm{R}=-0.825)$, butanedioic acid (Succinic acid) $(p=0.000$ and $\mathrm{R}=-0.891)$ and cellobiose $(p=0.000$ and $\mathrm{R}=-0.840$ ). Ruminiclostridium was negatively correlated with ethanedioc acid (Oxalic acid) $(p=0.000$ and $\mathrm{R}=-0.869)$ and $\mathrm{N}$-acetyl glucosamine $(p=0.000$ and $\mathrm{R}=-0.832)$. Further, two genera belonging to Prevotellaceae had a positive association with glycerol $(p=0.000$ and $\mathrm{R}=0.839)$ and rhamnose ( $p=0.000$ and $\mathrm{R}=0.788$ ). Alistepes had negative correlation with $\mathrm{N}$-acetyl glucosamine $(p=0.000$ and $\mathrm{R}=-0.832)$, ethanedioic acid $(p=0.000$ and $\mathrm{R}=-0.801)$ and benzene acetic acid $(p=0.000$ and $\mathrm{R}=-0.833)$. Helicobacter showed associations with hexadecanoic acid $(p=0.000$ and $\mathrm{R}=-0.801)$ and rhamnose $(p=0.000$ and $\mathrm{R}=0.792)$. Bacteriodes was positively correlated with galactose $(p=0.000$ and $\mathrm{R}=$ 0.803) (Fig. 4).

\section{Plasma endotoxin and histological study of intestine}

The level of endotoxin in CT group $(0.515662 \mathrm{EU} / \mathrm{ml})$ was higher than RB $(0.123426 \mathrm{Eu} / \mathrm{ml})$, IF $(0.073$ $\mathrm{EU} / \mathrm{ml})$ and $\mathrm{SF}(0.215 \mathrm{EU} / \mathrm{ml})$ groups $(p<0.05)$ (Fig. 5). HE stained intestine revealed the changes in enterocytes, villus length and crypt depth, lymphocytes infiltration and goblet cell distribution of the ileum in the treatment groups. Length of the villus was $169.85 \mu \mathrm{m}$ in the CT group with intact epithelia. The length reduced to $155.89 \mu \mathrm{m}$ and $151.84 \mu \mathrm{m}$ in the IF and RB treated groups and increased in SF treated group $(176.91 \mu \mathrm{m})$. Length of villus in all the treatment groups differed significantly compared to the controls. Moreover, the villus gap in the SF treated group was higher with respect to other treatment groups. The crypt depth in the CT group $(84.02 \mu \mathrm{m})$ had no significant difference with other treatment groups. The width of lamina propia and depth of sub mucosal layer were not altered among the treatment groups. Besides, width of lamina propria with much more infiltration of lymphocytic was observed in RB treated group followed by SF treated group. The number of goblet cells was comparatively higher in the SF treated group (Fig. 6).

\section{Discussion}

Although fermented foods and beverages have been known to impact the gastrointestinal well-being, their consumption is yet to be translated for global inclusion in world food guidelines (Bell et al. 2017). Though the research on the health benefits of fermented foods is relatively new, their consumption is becoming popular than ever before. Fermented foods and beverages have a significant share in the global food consumption contributing to one-third of the worldwide food intake under "functional foods" having enhanced nutritional aspects (Selhub et al. 2014). Furthermore, these nutrients and their association with the microbiota have also been reported to regulate our overall health (Bell et al. 2017). Microbes present in these foods help in the breakdown of the substrate during the fermentation by different mechanisms and exert probiotic properties on consumption. They also enhance the taste and 
other organoleptic properties which are very different from the starting substrate. On ingestion, the microbes pass through a hostile and stressful environment inside the digestive tract, viz. low pH of the stomach, digestive secretions like bile, pancreatin etc. Having passed these barriers, they stabilize and start colonizing mostly inside the colon. Though the time of their stay often called "persistence" is variable, they exert beneficial roles to the host in different aspects (Derrien and van Hylckama Vlieg 2015). These bacteria, mostly belonging to the category of Lactic Acid Bacteria from different fermented foods also modulate the overall health.

With a similar indication, the present study was conducted to demonstrate the effect of traditionally rice beverage on fecal microbes and metabolites in mice, using high throughput NGS technique and GC-MS analyses. Our research interests are on the claims on health modulating effects associated with its consumption by different communities of the region. It was hypothesized that the consumption of rice beverage might be associated with a change in the gut microbiota. Accordingly, mice were treated with the different extracts of rice beverage and compared its effect on gut microbiota. To the best of our knowledge, the present study would be the first to report on the effect of traditionally fermented rice beverage of North East India on the gut microbiota of mice.

The gut microbiota plays protective, metabolic and structural roles and is influenced by different factors, mostly by diet (Gentile and Weir 2018). Intake of different fractions of rice beverage during the treatment period did not interfere with the dietary intake and body weight gain mechanism. Therefore, no significant changes were observed in the food consumption and bodyweight of the animals. The alpha diversity indices did not change significantly except decrease in Pielou's evenness in the SF treatment. The upsurge of a dominant genus disturbs the overall diversity and abundance of others in an ecological niche. So, a decrease in Pielou's Evenness could have been resulted due to an increased abundance of Prevotellaceae UCG-001. Our results indicated a clear shift in the microbiota of all the treatment groups of which the RB treatment had a maximum change in 17 bacterial genera, 6 out of which belonged to the overall most abundant bacterial genera. The genera were Prevotellaceae UCG-001, Lactobacillus, Lachnospiraceae, Bacteroides, Alistipes and Helicobacter. This notable shift attracted our attention to the functionality of the major bacterial genera. Out of these bacteria, Bacteroides, Helicobacter and Alistipes were previously reported to be positively associated with intestinal inflammation (Bassett et al. 2015). Hence, a decrease in Bacteroides and Helicobacter could be a good indication. Furthermore, the bacteria known to promote healthy gut, viz. Muribaculaceae, Prevotellaceae, Ruminococcaceae, Candidatus saccharimonas were significantly increased in the RB treated group. An increased abundance of Muribaculaceae is associated with SCFA production and has a strong correlation with propionate in mice (Smith et al. 2019). Ruminococcaceae was previously reported to (i) enhance the immunity by promoting the gut barrier function, (ii) produce butyrate in the gut and (iii) associated inversely with the intestinal permeability (Madsen et al. 2001; Leclercq et al. 2014). An increase in Ruminococcaceae due to prebiotic treatment has also been reported (Shang et al. 2016). The SF treated group consumed relatively higher amounts of alcohol. In relevance to this, a decreased abundance of Ruminococcaceae in the SF treated group may be attributed as a detrimental effect of alcohol intake and is supported by a report in the mouse model (Bull-Otterson et al. 2013). Another study reported increased abundances of 
Lachnospiraceae, Helicobacter and Bacteroides in stressed mice compared with the normal group whereas these three genera contrarily decreased significantly in the RB treated group ( $L i$ et al. 2017). A similar trend was reported in animals fed with prebiotic, wherein Lachnospiraceae decreased and Ruminococcaceae increased significantly (Zhang et al. 2018). Therefore, it is an indication of prebiotic components in the rice beverage. Different species of Helicobacter are known to exert pathogenic effects on laboratory rodents (Ward et al. 1996). A decrease in its population is an indication of a healthy gut environment. Previously, Han et al. (2019) had reported a negative association between Helicobacter and Prevotella which supports this finding (Han et al. 2019). Increased abundance of Anaerotruncus was previously found to be linked with colorectal cancer (Candela et al. 2014). Another genus, Erysipelatoclostridium associated with metabolic syndrome, decreased in this study (Smith-Brown et al. 2016). A decreased incidence of Rodentibacter, the leading infectious agent in laboratory rodents indicated an improved immune function (Woting et al. 2014). In connection, Anaeroplasma, having protective effects against atherosclerosis increased in the RB treatment. The IF group had increased abundance of Christensenellaceae, Rikenellaceae and decreased Dorea which were previously reported in high fat diet fed animals (Woting et al. 2014). A relatively higher carbohydrate in the IF treated mice in the form of undissolved fermented residues compared to other treatments could have led to such changes. Nevertheless, Rikenellaceae has also been increased in prebiotic treated animals. Contrarily, the levels of Desulfovibrio another indicator of high fat diet was found to be lower in this treatment. Dietary differences have been associated with changes in the morphology of the small intestine leading to functional alterations in its structure. The goblet cells present in the intestinal epithelium produce several factors including trefoil factors and different mucins which form the mucus layer. Mucin 2 is the most abundant mucin and is associated with the organization of the intestinal mucous layers at the epithelial surface. This layer serves as a barrier to the pathogens restricting their entry towards the epithelial cells (Van der Sluis et al. 2006). An increased goblet cell number and hyperplasia in the SF treated group can be an indication of escalated mucin production due to the impact of alcohol as supported by earlier study (Rocco et al. 2014). Absorption and depletion of fatty acids and carbohydrates take place during the transit through the small intestine, hence the residing bacteria in the colon sustain by fermentation of the complex sugars (Donaldson et al. 2015). The soluble fraction contained relatively higher amount of alcohol along with soluble sugars which could elevate the triglyceride levels (Van De Wiel 2012). This might have resulted in an increase in the villous length in the SF treatment. Undigested fibers serve as energy source to the gut bacteria leading to the production of SCFAs which contribute to antiinflammatory effects. Relatively lower inter-villous gap and a lesser infiltration of polymorphonuclear cells (PMNCs) in lamina propria of the IF treated group indicates the anti-inflammatory effect. Similar physiological modifications due to dietary interventions were also reported earlier (Navarrete et al. 2015). Nevertheless, the presence of alcohol in the RB treatment may have counterpoised the effect and showed a prominent infiltration of PMNCs like the SF group, which might have resulted as inflammatory immune responses. Such responses are regulated by the cells present in Peyers' patches, lymphoid follicle or lamina propria. Observed features of these responses in SF and RB treatment could also be attributed to the increased abundance of Prevotellaceae in both the treatment groups. Species belonging to Prevotellaceae possess lipopolysaccharides (LPS), the most potent antigenic component of the Gram- 
negative bacterial cell wall. LPS is the most potent activators of innate immune signaling and an important mediator of the microbiome's influence on host physiology which is known to trigger inflammatory factors (Larsen 2017). We have earlier reported the presence of Hexadecanoic acid, a longchain fatty acid in rice based beverage which was fermented using a similar method in this study (Das et al. 2019). It is one of the flavor component and is abundant in rice wine from China (Yan et al. 2019). Interestingly, it was found to be present in the feces of all treatments and absent in the control, depicting its origin from the components of the beverage. Ruminococcus is a cellulolytic bacterium, possessing galactosidase enzyme and prefers to utilize cellobiose over glucose as a growth substrate (Cervera-Tison et al. 2012). Therefore, probably, Ruminococcaceae had a negative association with cellobiose and galactose. Succinic acid was reported to be associated with inflammation and dysbiosis and its negative association with Ruminococcaceae can also be regarded as a good indication for the RB treatment (Connors et al. 2019). Glycerol in feces results due to the hydrolysis of phospholipids by Prevotella. This supports the positive association of Prevotellaceae with glycerol in this study. Another metabolite, oxalic acid was detected in the feces of all treatments except RB and showed a negative correlation with Alistepes and Ruminiclostridium. Degradation of oxalic acid by probiotic bacteria is known to be an indicator of good renal health. There have been reports suggesting endotoxin reducing activity of fermented foods (Ahola et al. 2017). As expected, plasma endotoxins were found to be lowest in the IF followed by RB and SF treatments compared to the controls. Contrarily, the inflammation inducing effects as observed in histology of SF treated mouse intestine also had higher endotoxins level. Notably, the IF treatment, having no alcohol showed lowest endotoxin levels and the RB having a combination of IF and SF had intermediate endotoxin levels in the blood. Altogether, these results though preliminary indicated sundry effects of prebiotic and probiotic components of the rice beverage.

In summary, different fractions of the beverage affected the gut bacteria as well as metabolites differently. Rice beverage treated mice had healthier gut promoting bacteria. These results motivate further studies to explore the effect of rice beverage on gut microbial communities in a dose-dependent manner. In connection with the emergent reports on the health benefits of moderate alcohol consumption, this study further opens new dimensions for studying other aspects of consumption of this beverage (Caslin et al. 2019; Le Roy et al. 2020).

\section{Declarations}

\section{Acknowledgments}

Authors are thankful to Mrs. Anima Baishya for sharing traditional knowledge on rice beverage preparation, Mr. Gwhwm Basumatary and Mr. Abinash Nath for assistance in animal rearing and handling.

\section{Ethical statement}

All experiments were conducted following the guidelines of the Committee for the Purpose of Control and Supervision of Experiments on Animals (CPCSEA), Ministry of Environment, Forest and Climate change, 
Government of India and approved by institutional animal ethical committee (approval no. IASST/IAEC/2016-17/07). This article does not contain any studies with human participants performed by any of the authors.

\section{Funding}

This study was supported by the core fund of IASST and the Department of Biotechnology (DBT, Govt. of India) funded unit of excellence project (BT/550/NE/U-EXCEL/2014). The research was carried out in the Institutional Level Biotech Hub of IASST.

\section{Conflicts of interest}

The authors declare no conflicts of interest.

\section{Data availability statement}

The data that support the findings of this study are available on request from the corresponding author. Sequences are available via the MG-RAST under project ID mgp85071.

\section{Author contributions}

$\mathrm{BB}, \mathrm{AA}$, and MRK designed the research, $\mathrm{BB}$ and $\mathrm{AA}$ performed the experiments, SD and BB analyzed the NGS results. The manuscript was drafted by BB and revised by MRK.

\section{References}

Ahola AJ, Lassenius MI, Forsblom C, Harjutsalo V, Lehto M, Groop PH (2017) Dietary patterns reflecting healthy food choices are associated with lower serum LPS activity. Sci Rep 7:1-9 . https://doi.org/10.1038/s41598-017-06885-7

Balvočiute M, Huson DH (2017) SILVA, RDP, Greengenes, NCBI and OTT - how do these taxonomies compare? BMC Genomics 18(Suppl 2:1-8 . https://doi.org/10.1186/s12864-017-3501-4

Bassett SA, Young W, Barnett MPG, Cookson AL, McNabb WC, Roy NC (2015) Changes in composition of caecal microbiota associated with increased colon inflammation in interleukin-10 gene-deficient mice inoculated with Enterococcus species. Nutrients 7:1798-1816 . https://doi.org/10.3390/nu7031798

Bell V, Ferrão J, Fernandes T (2017) Nutritional Guidelines and Fermented Food Frameworks. Foods 6:65 . https://doi.org/10.3390/foods6080065

Bull-Otterson L, Feng W, Kirpich I, Wang Y, Qin X, Liu Y, Gobejishvili L, Joshi-Barve S, Ayvaz T, Petrosino J, Kong M, Barker D, McClain C, Barve S (2013) Metagenomic Analyses of Alcohol Induced Pathogenic Alterations in the Intestinal Microbiome and the Effect of Lactobacillus rhamnosus GG Treatment. PLoS One 8:e53028 . https://doi.org/10.1371/journal.pone.0053028 
Candela M, Turroni S, Biagi E, Carbonero F, Rampelli S, Fiorentini C, Brigidi P (2014) Inflammation and colorectal cancer, when microbiota-host mutualism breaks. World J Gastroenterol 20:908-922 . https://doi.org/10.3748/wjg.v20.i4.908

Caslin B, Maguire C, Karmakar A, Mohler K, Wylie D, Melamed E (2019) Alcohol shifts gut microbial networks and ameliorates a murine model of neuroinflammation in a sex-specific pattern. Proc Natl Acad Sci U S A 116:25808-25815 . https://doi.org/10.1073/pnas.1912359116

Cervera-Tison M, Tailford LE, Fuell C, Bruel L, Sulzenbacher G, Henrissat B, Berrin JG, Fons M, Giardina T, Juge N (2012) Functional analysis of family GH36 a-galactosidases from Ruminococcus gnavus E1: Insights into the metabolism of a plant oligosaccharide by a human gut symbiont. Appl Environ Microbiol 78:7720-7732 . https://doi.org/10.1128/AEM.01350-12

Chong J, Wishart DS, Xia J (2019) Using MetaboAnalyst 4.0 for Comprehensive and Integrative Metabolomics Data Analysis. Curr Protoc Bioinforma 68:86 . https://doi.org/10.1002/cpbi.86

Clemente JC, Ursell LK, Parfrey LW, Knight R (2012) The impact of the gut microbiota on human health: An integrative view. Cell 148:1258-1270 . https://doi.org/10.1016/j.cell.2012.01.035

Connors J, Dawe N, Van Limbergen J (2019) The role of succinate in the regulation of intestinal inflammation. Nutrients 11:1-12

Das AJ, Deka SC, Miyaji T (2012) Methodology of rice beer preparation and various plant materials used in starter culture preparation by some tribal communities of north-east india: A survey. Int Food Res $\mathrm{J}$ 19:101-107

Das AJ, Khawas P, Miyaji T, Deka SC (2014) HPLC and GC-MS analyses of organic acids, carbohydrates, amino acids and volatile aromatic compounds in some varieties of rice beer from northeast India. $J$ Inst Brew 120:244-252 . https://doi.org/10.1002/jib.134

Das S, Deb D, Adak A, Khan MR (2019) Exploring the microbiota and metabolites of traditional rice beer varieties of Assam and their functionalities. 3 Biotech 9:1-10 . https://doi.org/10.1007/s13205-019$1702-z$

Dehingia M, Thangjam K, Talukdar NC, Talukdar R, Reddy N, Mande SS, Deka M, Khan MR (2015) Gut bacterial diversity of the tribes of India and comparison with the worldwide data. Sci Rep 5:1-12 . https://doi.org/10.1038/srep18563

Derrien M, van Hylckama Vlieg JET (2015) Fate, activity, and impact of ingested bacteria within the human gut microbiota. Trends Microbiol. 23:354-366

Donaldson GP, Lee SM, Mazmanian SK (2015) Gut biogeography of the bacterial microbiota. Nat. Rev. Microbiol. 14:20-32 
Foster KR, Schluter J, Coyte KZ, Rakoff-Nahoum S (2017) The evolution of the host microbiome as an ecosystem on a leash. Nature 548:43-51

Gentile CL, Weir TL (2018) The gut microbiota at the intersection of diet and human health. Science (80-) $362: 776-780$

Ghosh K, Ray M, Adak A, Dey P, Halder SK, Das A, Jana A, Parua S, Das Mohapatra PK, Pati BR, Mondal KC (2015) Microbial, saccharifying and antioxidant properties of an Indian rice based fermented beverage. Food Chem 168:196-202 . https://doi.org/10.1016/j.foodchem.2014.07.042

Guarner F, Malagelada JR (2003) Gut flora in health and disease. Lancet 361:512-519 . https://doi.org/10.1016/S0140-6736(03)12489-0

Han H, Lee S-Y, Oh S, Moon H, Cho H, Kim J-H (2019) Correlations of the Gastric and Duodenal Microbiota with Histological, Endoscopic, and Symptomatic Gastritis. J Clin Med 8:312 .

https://doi.org/10.3390/jcm8030312

Jeyaram K, Mohendro Singh W, Premarani T, Devi AR, Chanu KS, Talukdar NC, Singh MR (2008) Molecular identification of dominant microflora associated with "Hawaijar" - A traditional fermented soybean (Glycine max (L.)) food of Manipur, India. Int J Food Microbiol 122:259-268 . https://doi.org/10.1016/j.jfoodmicro.2007.12.026

Katoh K, Standley DM (2013) MAFFT multiple sequence alignment software version 7: Improvements in performance and usability. Mol Biol Evol 30:772-780 . https://doi.org/10.1093/molbev/mst010

Khan, M.R., Bhaskar B, AdaK, A., Talukdar NC (2017) Rice based beverage with high alcohol content and method therefor

Larsen JM (2017) The immune response to Prevotella bacteria in chronic inflammatory disease. Immunology 151:363-374 . https://doi.org/10.1111/imm.12760

Le Roy Cl, Wells PM, Si J, Raes J, Bell JT, Spector TD (2020) Red Wine Consumption Associated With Increased Gut Microbiota a-Diversity in 3 Independent Cohorts. Gastroenterology 158:270-272.e2 . https://doi.org/10.1053/j.gastro.2019.08.024

Leclercq S, Matamoros S, Cani PD, Neyrinck AM, Jamar F, Stärkel P, Windey K, Tremaroli V, Bäckhed F, Verbeke K, De Timary P, Delzenne NM (2014) Intestinal permeability, gut-bacterial dysbiosis, and behavioral markers of alcohol-dependence severity. Proc Natl Acad Sci U S A 111:E4485-E4493 . https://doi.org/10.1073/pnas.1415174111

Li S, Wang Z, Yang Y, Yang S, Yao C, Liu K, Cui S, Zou Q, Sun H, Guo G (2017) Lachnospiraceae shift in the microbial community of mice faecal sample effects on water immersion restraint stress. AMB Express 7:82 . https://doi.org/10.1186/s13568-017-0383-4 
Madsen K, Cornish A, Soper P, McKaigney C, Jijon H, Yachimec C, Doyle J, Jewell L, De Simone C (2001) Probiotic bacteria enhance murine and human intestinal epithelial barrier function. Gastroenterology 121:580-591 . https://doi.org/10.1053/gast.2001.27224

Marco ML, Heeney D, Binda S, Cifelli CJ, Cotter PD, Foligné B, Gänzle M, Kort R, Pasin G, Pihlanto A, Smid EJ, Hutkins R (2017) Health benefits of fermented foods: microbiota and beyond. Curr Opin Biotechnol 44:94-102 . https://doi.org/10.1016/j.copbio.2016.11.010

Navarrete J, Vásquez B, del Sol M (2015) Morphoquantitative analysis of the lleum of C57BL/6 mice (Mus musculus) fed with a high-fat diet. Int J Clin Exp Pathol 8:

Rocco A, Compare D, Angrisani D, Sanduzzi Zamparelli M, Nardone G (2014) Alcoholic disease: Liver and beyond. World J. Gastroenterol. 20:14652-14659

Roy A, Khanra K, Mishra A, Bhattacharyya N (2012) General analysis and Antioxidant study of Traditional fermented drink Handia, its concentrate and volatiles. Adv Life Sci its Appl 1:54-57

Selhub EM, Logan AC, Bested AC (2014) Fermented foods, microbiota, and mental health: Ancient practice meets nutritional psychiatry. J Physiol Anthropol 33:1-12 . https://doi.org/10.1186/1880-680533-2

Shang Q, Shan X, Cai C, Hao J, Li G, Yu G (2016) Dietary fucoidan modulates the gut microbiota in mice by increasing the abundance of: Lactobacillus and Ruminococcaceae. Food Funct 7:3224-3232 . https://doi.org/10.1039/c6fo00309e

Shibayama J, Kuda T, Shikano A, Fukunaga M, Takahashi H, Kimura B, Ishizaki S (2018) Food Bioscience $E$ ff ects of rice bran and fermented rice bran suspensions on caecal microbiota in dextran sodium sulphate-induced in fl ammatory bowel disease model mice. Food Biosci 25:8-14 . https://doi.org/10.1016/j.fbio.2018.07.002

Smith-Brown P, Morrison M, Krause L, Davies PSW (2016) Dairy and plant based food intakes are associated with altered faecal microbiota in 2 to 3 year old Australian children. Sci Rep 6:1-8 . https://doi.org/10.1038/srep32385

Smith BJ, Miller RA, Ericsson AC, Harrison DC, Strong R, Schmidt TM (2019) Changes in the gut microbiome and fermentation products concurrent with enhanced longevity in acarbose-treated mice. BMC Microbiol 19:1-16. https://doi.org/10.1186/s12866-019-1494-7

Van De Wiel A (2012) The effect of alcohol on postprandial and fasting triglycerides. Int J Vasc Med 2012:1-4 . https://doi.org/10.1155/2012/862504

Van der Sluis M, De Koning BAE, De Bruijn ACJM, Velcich A, Meijerink JPP, Van Goudoever JB, Büller HA, Dekker J, Van Seuningen I, Renes IB, Einerhand AWC (2006) Muc2-Deficient Mice Spontaneously Develop 
Colitis, Indicating That MUC2 Is Critical for Colonic Protection. Gastroenterology 131:117-129 . https://doi.org/10.1053/j.gastro.2006.04.020

Ward JM, Anver MR, Haines DC, Melhorn JM, Gorelick P, Yan L, Fox JG (1996) Inflammatory large bowel disease in immunodeficient mice naturally infected with Helicobacter hepaticus. Lab Anim Sci 46:15-20

Wei T, Simko V, Levy M, Xie Y, Jin Y, Zemla J (2017) Package 'corrplot.' Statistician 56:e24

Weir TL, Manter DK, Sheflin AM, Barnett BA, Heuberger AL, Ryan EP (2013) Stool Microbiome and Metabolome Differences between Colorectal Cancer Patients and Healthy Adults. PLoS One. https://doi.org/10.1371/journal.pone.0070803

Woting A, Pfeiffer N, Loh G, Klaus S, Blaut M (2014) Clostridium ramosum promotes High-Fat dietinduced obesity in Gnotobiotic Mouse Models. MBio 5:1-10 . https://doi.org/10.1128/mBio.01530-14

Yan S, Xiangsong C, Xiang X (2019) Improvement of the aroma of lily rice wine by using aroma-producing yeast strain Wickerhamomyces anomalus HN006. AMB Express 9:89 . https://doi.org/10.1186/s13568019-0811-8

Zhang Z, Wang X, Han S, Liu C, Liu F (2018) Effect of two seaweed polysaccharides on intestinal microbiota in mice evaluated by illumina PE250 sequencing. Int J Biol Macromol 112:796-802 . https://doi.org/10.1016/j.jjbiomac.2018.01.192

Zhou QQ, Yang DZ, Luo YJ, Li SZ, Liu FY, Wang GS (2011) Over-starvation aggravates intestinal injury and promotes bacterial and endotoxin translocation under high-altitude hypoxic environment. World $\mathrm{J}$ Gastroenterol 17:1584-1593 . https://doi.org/10.3748/wjg.v17.i12.1584

\section{Tables}

Table 1 Nutrient composition of the rodent diet

\begin{tabular}{|ll|}
\hline Nutrients & Content $(\mathrm{g} / \mathrm{kg})$ \\
\hline Crude protein & $>1.8$ \\
Crude fat & $>0.3$ \\
Crude fiber & $>0.7$ \\
\hline Ash & $<0.7$ \\
\hline
\end{tabular}

Table 2 Alpha diversity indices of gut microbiota of mice treated with different fractions of rice beverage. 


\begin{tabular}{|lllllllll|}
\hline Sample* $^{*}$ & \multicolumn{2}{l}{ Shannon index } & \multicolumn{2}{l}{ Faith PD } & \multicolumn{2}{l|}{ Pielou's Evenness } & \multicolumn{2}{l|}{ Observed OTUs } \\
\cline { 2 - 9 } & $\begin{array}{l}\mathbf{0}^{\text {th }} \\
\text { day }\end{array}$ & $\begin{array}{l}\mathbf{3 0}^{\text {th }} \\
\text { day }\end{array}$ & $\begin{array}{l}\mathbf{0}^{\text {th }} \\
\text { day }\end{array}$ & $\begin{array}{l}\mathbf{3 0}^{\text {th }} \\
\text { day }\end{array}$ & $\begin{array}{l}\mathbf{0}^{\text {th }} \\
\text { day }\end{array}$ & $\begin{array}{l}\mathbf{3}^{\text {th }} \\
\text { day }\end{array}$ & $0^{\text {th }}$ day & $\begin{array}{l}\mathbf{3 0}^{\text {th }} \\
\text { day }\end{array}$ \\
\hline CT & 8.56 & 8.42 & 78.16 & 97.24 & 0.86 & 0.85 & 1026.00 & 970.00 \\
\hline RB & 8.61 & 8.37 & 78.74 & 71.01 & 0.86 & 0.86 & 1011.67 & 827.67 \\
\hline IF & 8.37 & 8.28 & 77.97 & 81.35 & 0.85 & 0.85 & 924.33 & 844.33 \\
\hline SF & 8.70 & 8.18 & 93.17 & 87.43 & 0.87 & $0.83^{*}$ & 1061.00 & 954.67 \\
\hline
\end{tabular}

*(CT= controls, $\mathrm{RB}=$ rice beverage, $\mathrm{IF}=$ insoluble fraction and $\mathrm{SF}=$ soluble fraction $)$

Table 3 Relative abundance of bacteria after 30 days of treatment. 


\begin{tabular}{|c|c|c|c|}
\hline Treatment* & Bacteria & $\begin{array}{l}\text { Percent } \\
\text { abundance } \\
\text { ( } 0^{\text {th }} \text { day) }\end{array}$ & $\begin{array}{l}\text { Percent } \\
\text { abundance } \\
\text { (30 day) }\end{array}$ \\
\hline \multirow{17}{*}{ RB } & Bacteroides & 7.95 & 3.37 \\
\hline & Muribaculaceae & 0.5 & 1.54 \\
\hline & Prevotellaceae UCG-001 & 1.7 & 14.05 \\
\hline & Alistipes & 4.7 & 6.92 \\
\hline & Helicobacter & 4.95 & 3.02 \\
\hline & Lactobacillus & 6.05 & 0.23 \\
\hline & Clostridiales D_4_Family XII & 0.07 & 0.005 \\
\hline & Lachnoclostridium & 0.63 & 0.08 \\
\hline & Lachnospiraceae & 9.59 & 6.26 \\
\hline & Anaerotruncus & 0.35 & 0.07 \\
\hline & Ruminococcaceae NK4A214 group & 0.08 & 0.39 \\
\hline & Ruminococcaceae D_5_UBA1819 & 0 & 0.329 \\
\hline & Erysipelatoclostridium & 0.68 & 0.06 \\
\hline & Candidatus saccharimonas & 0.82 & 1.62 \\
\hline & Betaproteobacteriale & 0.06 & 0.007 \\
\hline & Rodentibacter & 0.74 & 0.01 \\
\hline & Anaeroplasma & 0.003 & 0.07 \\
\hline \multirow{7}{*}{ IF } & $\begin{array}{l}\text { Muribaculaceae D_5_uncultured } \\
\text { bacterium }\end{array}$ & 6.56 & 3.96 \\
\hline & Prevotellaceae & 0.64 & 0.39 \\
\hline & Rikenellaceae & 0.03 & 0.39 \\
\hline & Christensenellaceae R-7 group & 0 & 0.03 \\
\hline & Dorea & 0.01 & 0 \\
\hline & Lachnoclostridium & 0.88 & 0.18 \\
\hline & Desulfovibrio & 0.007 & 0 \\
\hline \multirow[t]{2}{*}{ SF } & Bacteroides & 7.00 & 3.70 \\
\hline & Muribaculaceae & 1.00 & 0.14 \\
\hline
\end{tabular}




\begin{tabular}{|lll|} 
Prevotellaceae UCG-001 & 7.93 & 17.79 \\
\hline Alistipes & 4.82 & 2.54 \\
\hline Clostridiales vadin BB60 group & 0.033 & 0.007 \\
\hline Ruminococcaceae UCG-014 & 4.38 & 2.33 \\
\hline Leptotrichia & 0.06 & 0.01 \\
\hline Desulfovibrionaceae & 0.16 & 0.08 \\
\hline Mycoplasma & 0.657 & 1.32 \\
\hline
\end{tabular}

*(CT= control, $\mathrm{RB}=$ rice beverage, $\mathrm{IF}=$ insoluble fraction and $\mathrm{SF}=$ soluble fraction $)$

\section{Figures}

(a)
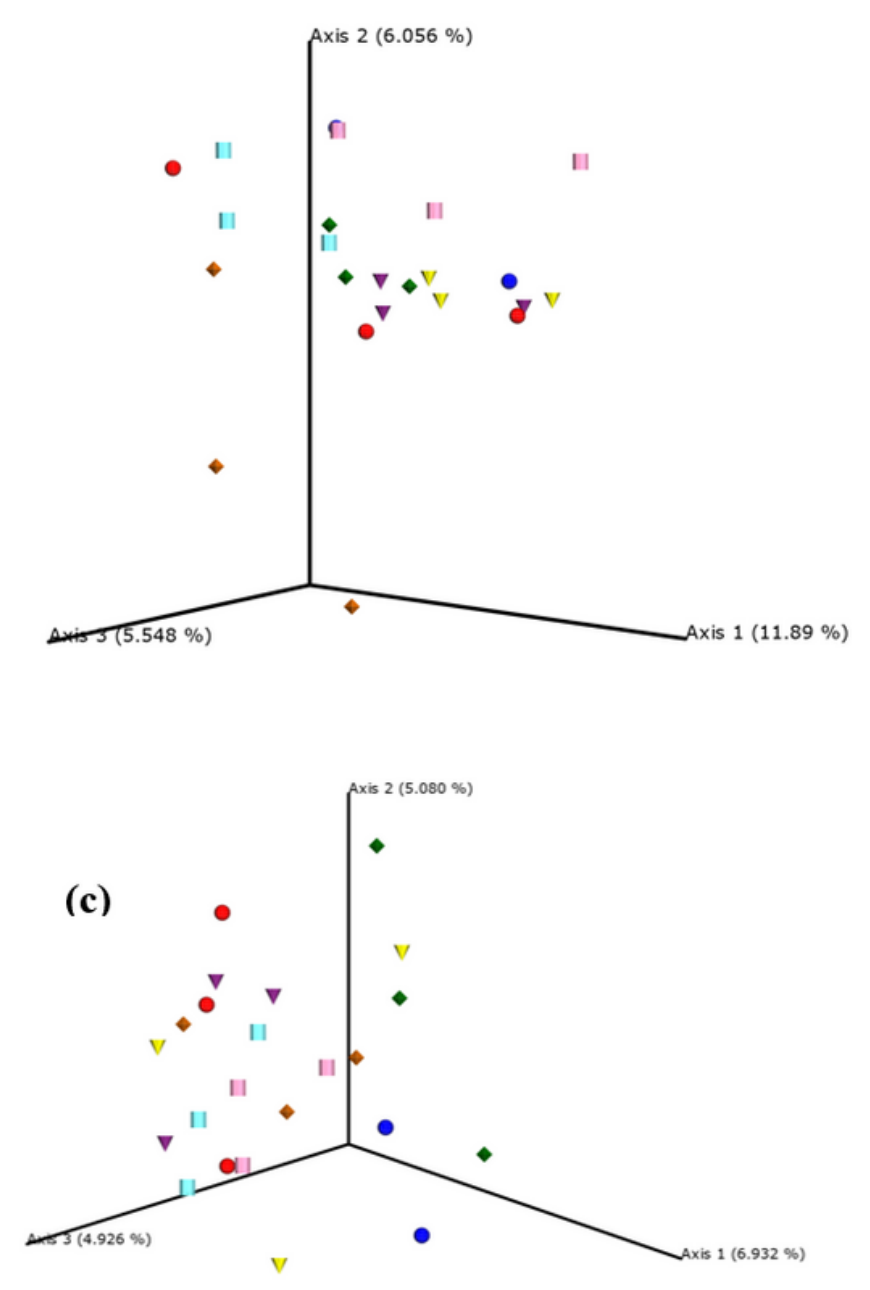

(b)
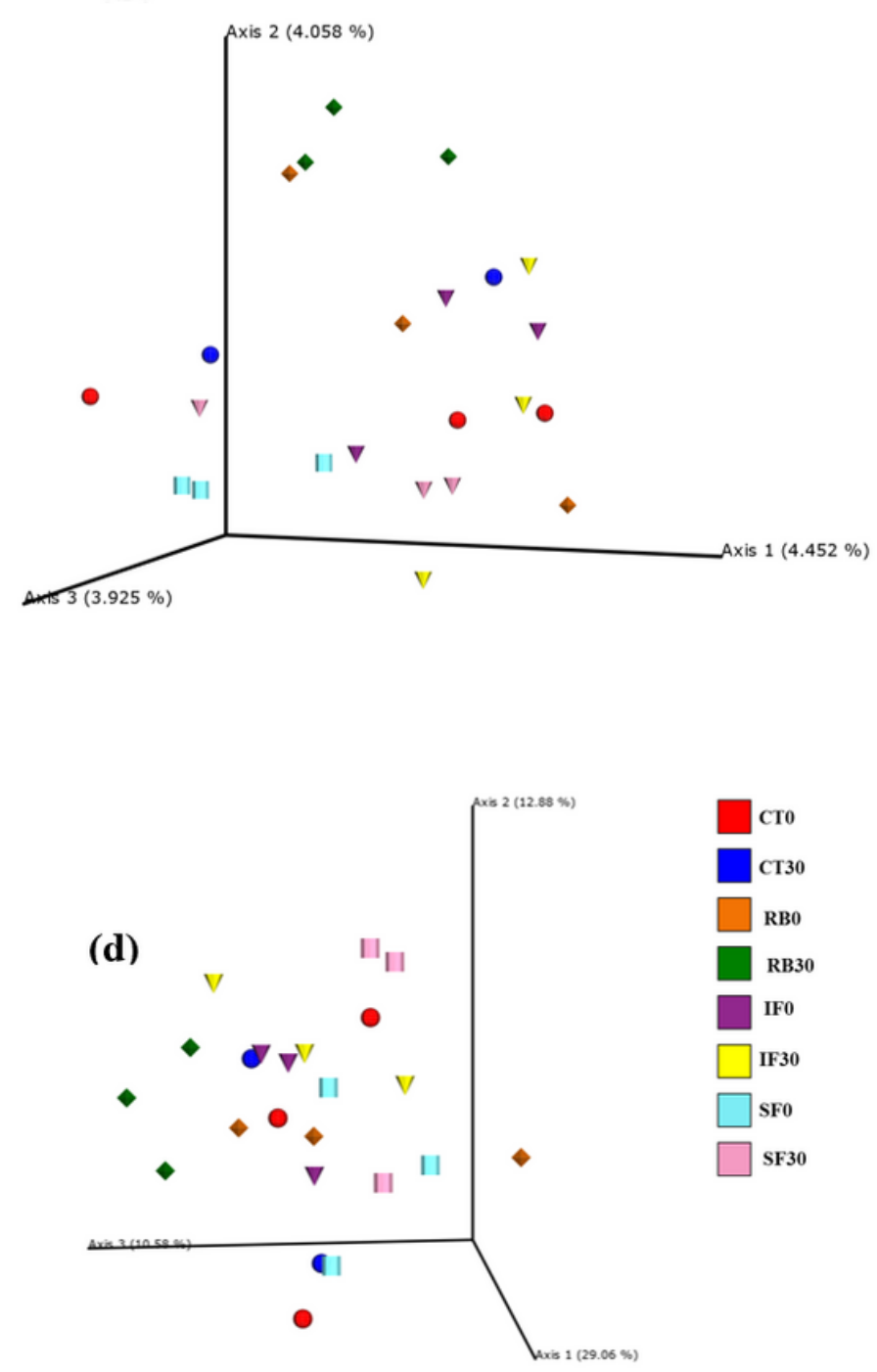

Figure 1 
Principal coordinate analysis (PCoA) showing beta diversity indices. (a) Bray Curtis, (b) Jaccard's, (c) Unweighted UniFrac and (d) Weighted UniFrac indices on bacterial diversity of fecal metagenomic DNA of mice treated with different fractions of rice beverage.

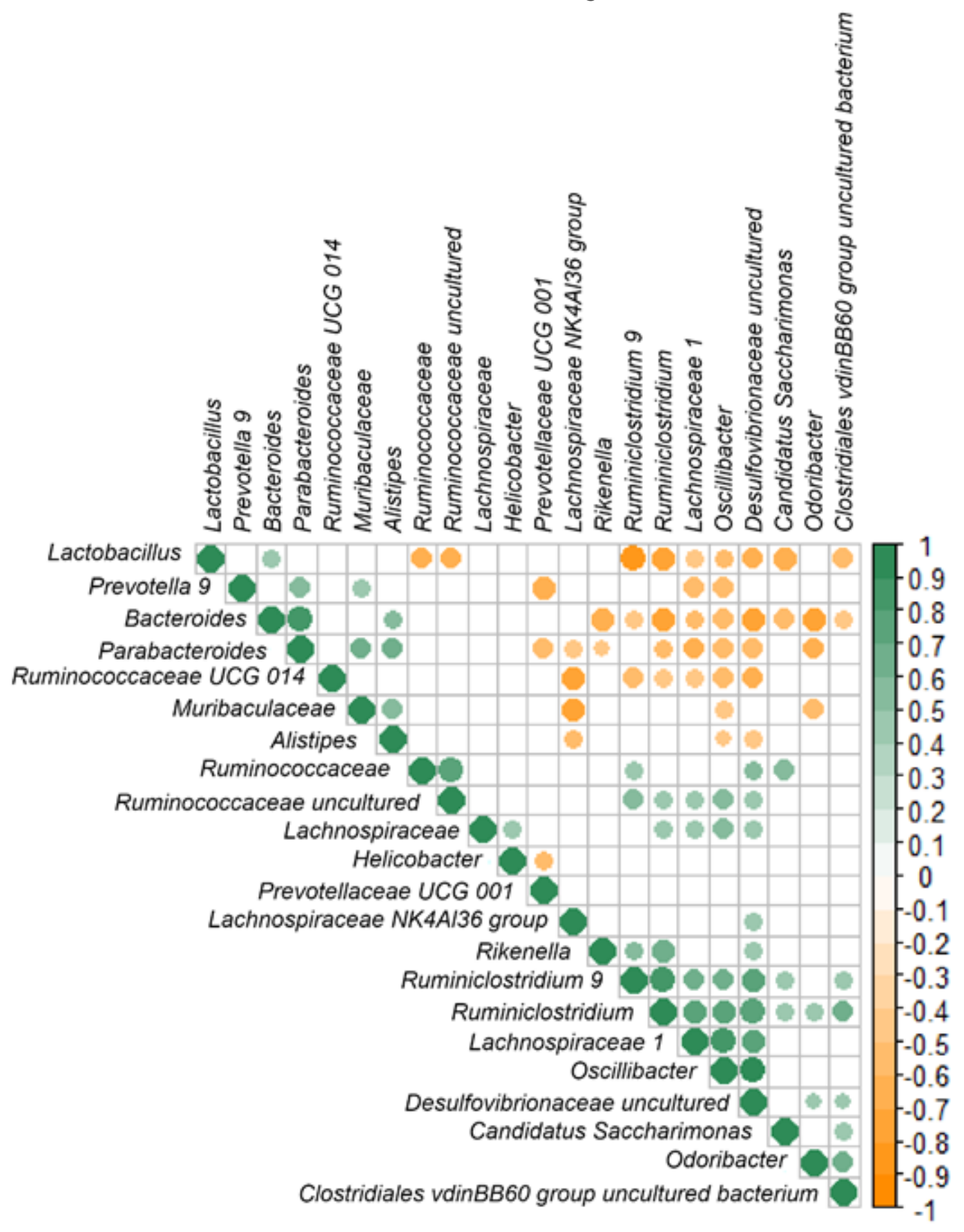

Figure 2

Correlation matrix among the 22 differently abundant bacterial genera. The Spearman's rank correlation coefficients ranged from 1.0 to -1.0 , corresponding to a strongly positive to a strongly negative correlations and has been represented in hierarchal order in the correlation plot. 


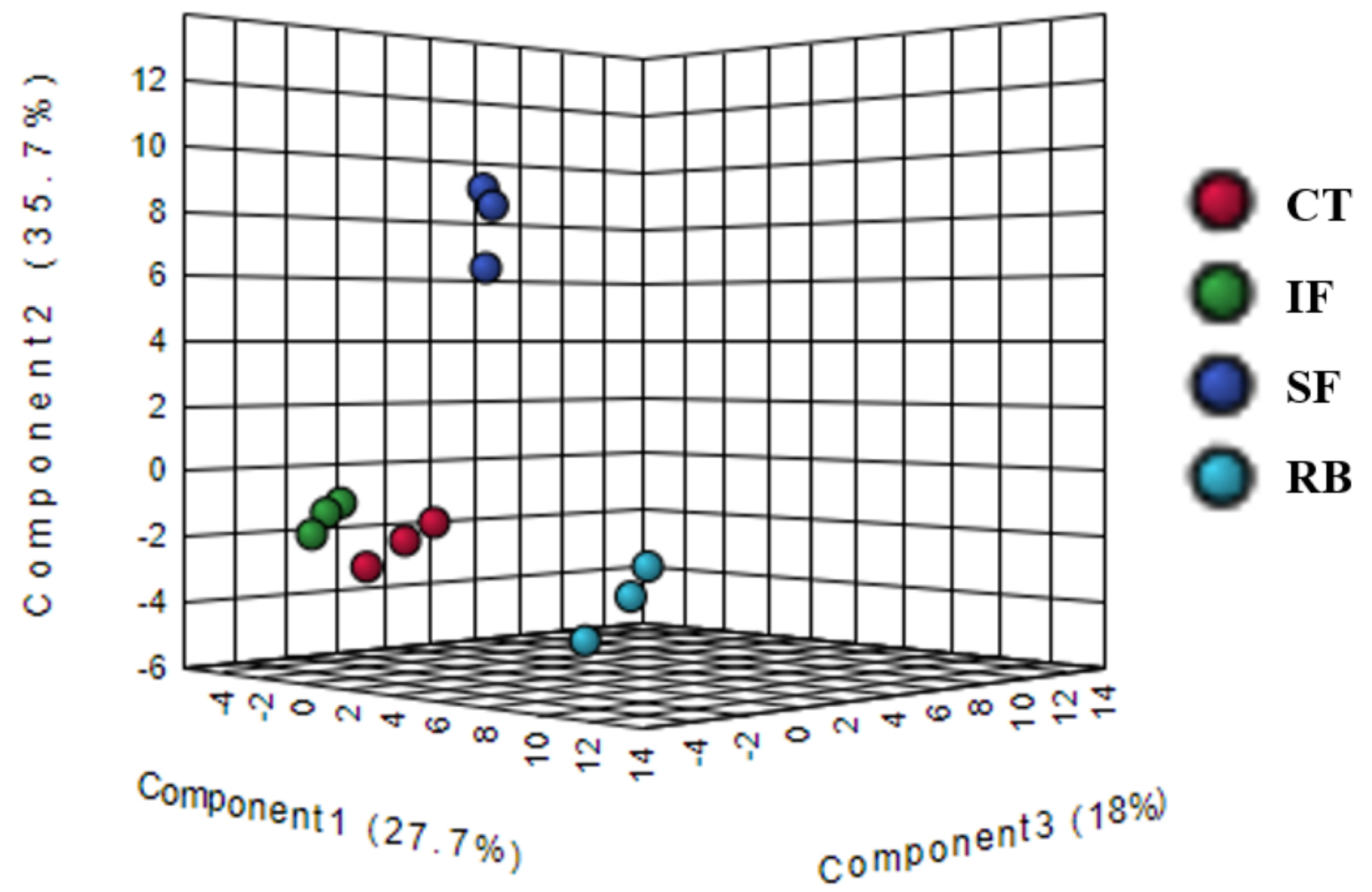

Figure 3

Partial least square discriminant analysis (PLS-DA) plot based on the metabolites detected in the feces of different treatment groups $(\mathrm{CT}=$ control, $\mathrm{RB}=$ rice beverage, $\mathrm{IF}=$ insoluble fraction and $\mathrm{SF}=$ soluble fraction). 


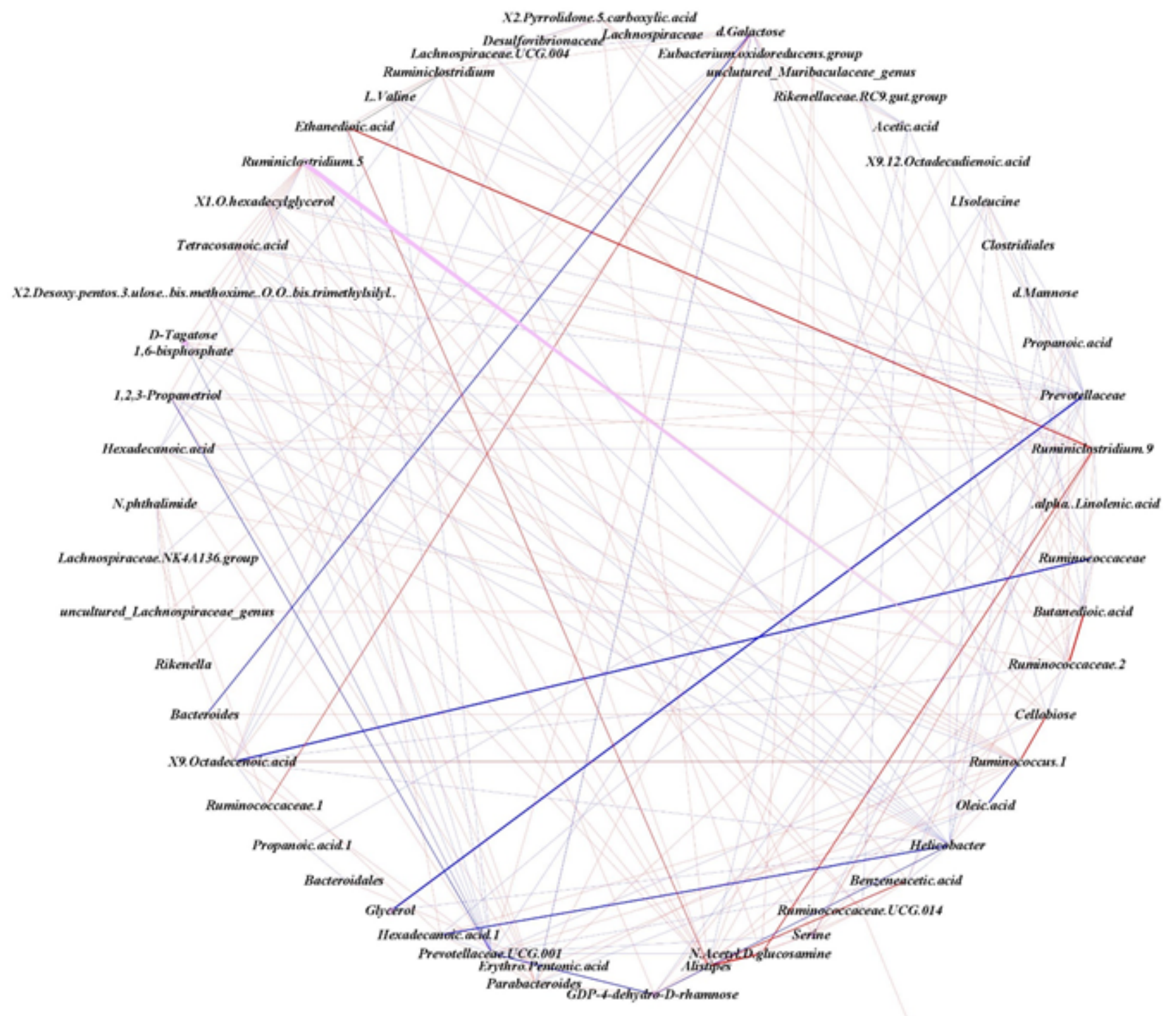

\section{Figure 4}

Network analysis showing the linkages of core bacterial genera with metabolites. Blue and red branches connecting the nodes indicate positive and negative correlations, respectively. Color intensities and branch thicknesses of the nodes reflect the degree of correlations. 


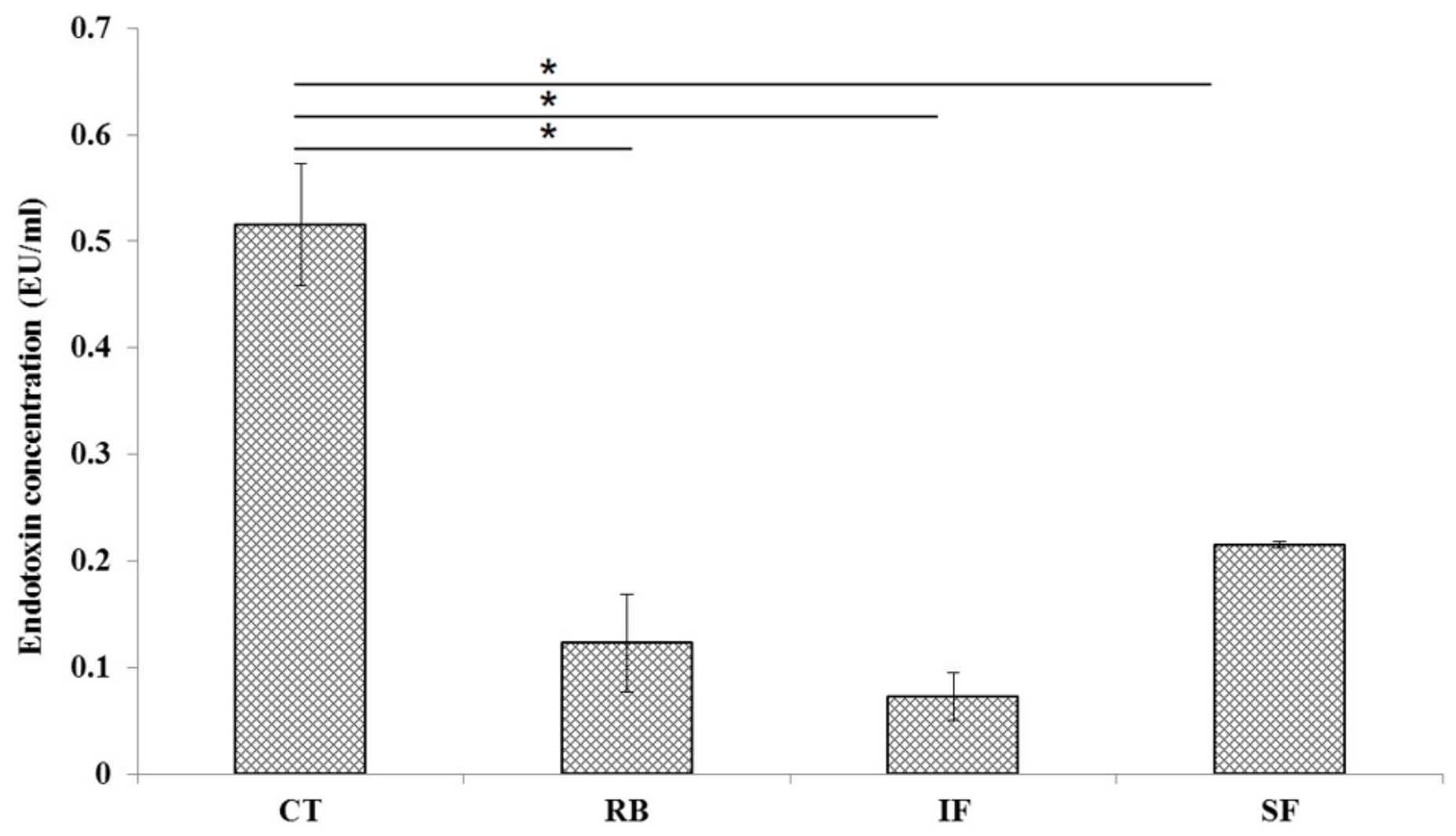

Figure 5

Endotoxin level in the blood plasma of the different groups after 30 days of treatment with different fractions of rice beverage. $(\mathrm{CT}=$ control, $\mathrm{RB}=$ rice beverage, $\mathrm{IF}=$ insoluble fraction and $\mathrm{SF}=$ soluble fraction).
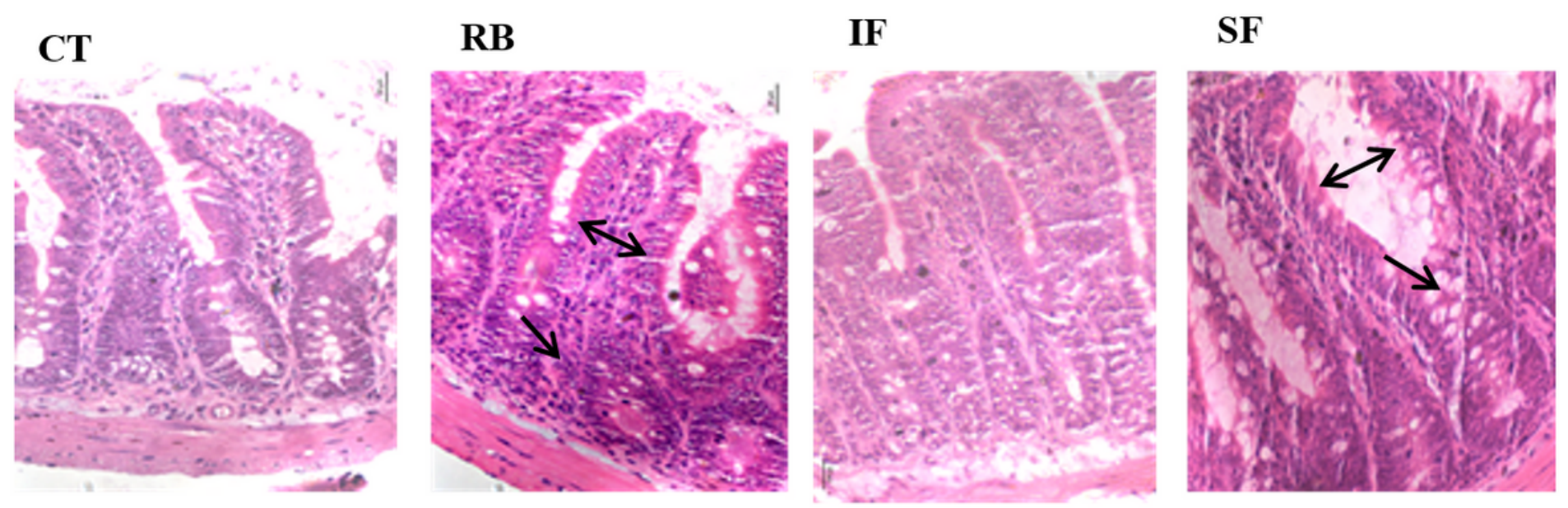

\section{Figure 6}

Light microscopic images of ileum of mice in the treatment groups (CT= control, RB= rice beverage, IF= insoluble fraction and SF= soluble fraction). Intact epithelia and ordered crypts in CT group, distorted crypts and higher infiltration of lymphocytes and wider lamina propria in the RB group. Lower number of 
goblet cells, lower villus gap and less lymphocytes in lamina propia were observed in the IF group. Higher number of goblet cells to the apical surface with loss of storage granules and higher villus gap observed in the SF group by HE staining after 30 days of treatment. 